\title{
Leaders
}

\section{What's new in the diagnosis of head injury?}

\author{
J F Geddes
}

Fatal head injury is commonly encountered by pathologists whose practice includes coroner's postmortem examinations. In most cases a macroscopic diagnosis of "head injury" can be given as the cause of death, and further examination of the brain is the province of those who are interested in the detailed neuropathology of craniospinal trauma. However, there are occasional cases in which, despite a history of head trauma and subsequent coma or vegetative state, no obvious cause of death is seen on external examination of the brain. Such patients are found to suffer from diffuse brain pathology of various types, which can only be diagnosed histologically, and which in most cases turn out to be either diffuse axonal damage or diffuse hypoxic damage, more commonly the former. When attempting to assess the extent of a head injury it is important to appreciate that axonal damage is triggered directly by head injury, but hypoxic damage is caused by secondary insults - hypotension, respiratory difficultiesoccurring in the immediate post-traumatic period. The distinction between axonal injury and hypoxic damage may be important, particularly when it may subsequently be used as evidence in a court of law.

Recent advances in the understanding of the subcellular pathology of traumatic white matter damage have led to the development of antisera to detect axonal injury, which together with immunostains for microglia have now superseded the traditional silver, cresyl-fast violet, and Marchi stains in routine head injury diagnosis. Awareness of the diagnostic pitfalls created by these new techniques, particularly in the detection of early pathological changes, is essential.

Department of Morbid

Anatomy and

Histopathology, St

Bartholomew's and the

Royal London School

of Medicine and

Dentistry, London,

United Kingdom

J F Geddes

Correspondence to: Dr Geddes,

Department of Morbid Anatomy, Royal London Hospital, Whitechapel,

London E1 1BB, United

Kingdom.

Accepted for publication 25 February 1997 spectrum, a damaged axon may show no than mild temporary transmembrane ionic disturbances, which reverse spontaneously. ${ }^{3}$ With more severe injury, morphological studies in humans have shown that traumatic axonal injury is an evolving process of reactive change, ${ }^{45}$ which is attributed to progressive damage to structural elements in the axonal cytoskeleton, demonstrated repeatedly in experimental studies. ${ }^{6}$ Despite the fact that animal models may not exactly replicate the process described in humans, either in distribution of lesions or in the time course of posttraumatic events, they are widely used to simulate human axonal injury to shed light on mechanisms of damage. ${ }^{78}$ These models show a common response by axons to non-disruptive mechanical deformation, with resultant ultrastructural changes, detectable within a few minutes of injury. ${ }^{910}$ The first change is swelling of mitochondria followed by focal increases in axonal diameter. Damage to microtubules, which become misaligned, follows rapidly. ${ }^{112}$ As structural integrity of microtubules is essential for normal anterograde and retrograde axonal flow, ${ }^{13}$ any change in their arrangement will interfere with normal transport of organelles down the axon. If the injury is severe, the neurofilament backbone of the axoplasm also collapses, neurofilament sidearms are lost, and the cytoskeleton disintegrates at the point of damage. The axolemma begins to fold inwards, and separate from the overlying myelin sheath. This damage is localised, however, so that proteins continue to be transported down the axon until they reach the site of injury, where they accumulate because they are unable to progress further. A swelling or axonal "bulb" of accumulated membranous organelles enlarges upstream of the site of injury over the next few hours; eventually, irreversible axotomy takes place. ${ }^{12}$ This is not a single event occurring at a precise time after injury; it is now believed that continued recruitment of damaged axons takes place, and that the process leading to secondary axotomy continues over a longer time scale than has previously been thought. ${ }^{14}$ Understanding of the evolving nature of reactive axonal change has led to the concept of a "therapeutic window" for diffuse axonal injury, during which neuroprotective agents would have a role-at present only theoretical-in minimising the sequelae of trauma.

What initiates the potentially catastrophic sequence of cellular events in axonal injury? Although the exact mechanism is uncertain, there is increasing evidence that the integrity of the axolemmal membrane is disturbed by 
mechanical strain $^{412}$ that renders it leaky, probably for prolonged periods. ${ }^{15}$ More than one author has speculated that calcium influx may be responsible for collapse of structural elements in the axon. A recent study of stretch injury in an optic nerve model has provided the first cytochemical evidence of intra-axonal calcium accumulation and redistribution of calcium membrane pump $\mathrm{Ca}$-ATPase and ecto$\mathrm{Ca}-\mathrm{ATPase}$ activity, together with increased permeability of the axolemma, ${ }^{9}$ suggesting that this may indeed be the mechanism involved.

\section{Sampling for diffuse traumatic brain damage}

Diffuse axonal injury (DAI) is a diagnosis that may be suspected either radiologically ${ }^{16}$ or from the macroscopic appearances of the sliced brain using well established criteria. ${ }^{17}$ It can only be confirmed, however, by looking at sections of blocks taken from several areas of the brain, above and below the tentorium. The definition of DAI is multifocal traumatic axonal damage in cerebral hemispheres, corpus callosum, and brain stem, with variable involvement of the cerebellum. ${ }^{18}$ Could one perhaps make the diagnosis with three blocks from these areas? We have recently tried a technique for screening for DAI in 22 cases, previously diagnosed with blocks from all areas of the brain. We looked at three standard blocks from sites above and below the tentorium, to assess the presence of traumatic axonal damage. We found that restricted sampling, even from areas known to be vulnerable to traumatic axonal injury, would have led to an underestimation of the pathology in nine of the cases; therefore, diffuse brain damage can only be assessed using a larger, more representative series of blocks. ${ }^{19}$ In light of this experience, it seems that a minimum set of samples should include: two blocks of corpus callosum (one anterior and one posterior) together with parasagittal white matter; one block of the posterior limb of the internal capsule; one block of cerebellar hemispheric white matter with the middle cerebellar peduncle; and at least one of the upper brain stem, ideally including the superior cerebellar peduncle. This sampling scheme would include the sites most vulnerable to traumatic damage and minimise the chance of under or overdiagnosing the condition.

Routine and immunohistochemical stains The panel of routine and immunohistochemical stains used for diagnosis by neuropathologists interested in head injury usually comprises haematoxylin and eosin, $\beta$ amyloid precursor protein ( $\beta \mathrm{APP})$, a microglial marker such as CD68/PG-M1, and glial fibrillary acidic protein (GFAP). $\beta A P P$ is a glycoprotein synthesised in the neuronal cytoplasm which is transported down the axon by fast anterograde transport. Normally present in axons at undetectable levels, it accumulates where axonal transport is disturbed, and can then be used as a marker of axonal injury, although it is not specific for trauma. $\beta$ APP immunocytochemistry permits identification of axonal damage as early as two to three hours after head injury ${ }^{20-23}$ -in one study in less than two hours. ${ }^{24}$ The antiserum produces a clean picture of damaged axons staining against a background of negatively stained normal brain, which makes screening of large areas of brain relatively simple. Linear positivity of axons is the earliest change, but with longer survival of about five hours, reactive axonal varicosities and bulb formation can be detected, well before changes are detectable by haematoxylin and eosin or the traditional silver stains (approximately 18 to 24 hours' survival time ${ }^{25}$ ). With increasing survival times, swellings which were initially positive no longer stain, although the time at which positivity disappears may vary in different parts of the same brain. With prolonged survival (longer than one year) occasional granular extracellular deposits of $\beta A P P$ are the only abnormality detectable with this immunostain. ${ }^{19}$

With the arrival of reliable immunocytochemical methods to demonstrate microglial associated antigens, it has become clear that there is an early microglial response to axonal damage. ${ }^{26}$ Immunoperoxidase staining to the PG-M1 epitope of CD68 demonstrates progressive microglial hypertrophy and proliferation in damaged areas of white matter from 24 hours' survival following head injury, and microglial clusters forming around axonal bulbs from about two weeks' survival. ${ }^{19}$ With several months' survival, the presence of microglia and sheets of foamy macrophages in an anatomical distribution in the ascending and descending long tracts indicates Wallerian degeneration secondary to the original axonal damage.

Immunocytochemistry for GFAP shows no specific features. In the brains of long term survivors of traumatic axonal damage, in whom microglial nodules have long since disappeared, cerebral atrophy and diffuse astrocytic gliosis of the white matter are the only residua. GFAP used in conjunction with haematoxylin and eosin and PG-M1 stains is, however, a useful way of screening for significant hypoxic brain damage that may accompany DAI. The injured brain is extremely sensitive to hypoxic insults, ${ }^{12}$ and secondary hypoxic brain damage is common in severe head injury. ${ }^{27}{ }^{28}$ Established nerve cell damage and loss is invariably accompanied by increased microglial and astrocytic activity, and as these cells are not seen in great numbers in normal neocortex or deep grey matter, any increase is likely to represent a pathological change, which may need further investigation. Under 24 hours haematoxylin and eosin stain remains the most reliable way of detecting ischaemic neuronal change.

\section{Possible pitfalls in diagnosis}

The ability to detect axonal damage immunocytochemically at a time when all other stains are negative may give rise to a number of interpretative problems. Accumulation of $\beta A P P$ in axons indicates interruption to the process of fast axonal transport, but it is not always easy to know what significance to place on the finding of positively staining axons - that is, how severe 
the original injury was, if such staining is present before swellings have fully formed and therefore before secondary axotomy could have occurred. Might these axons have suffered mild, temporary damage that would have reversed had the patient lived? It is not impossible, given the current belief that a spectrum of severity of axonal damage occurs, some of it reversible. $^{329}$ Once large swellings have formed, however, between 18 and 24 hours' survival, the likelihood is that they represent permanent damage. Immunostaining for $\beta A P P$ is a reliable marker of axonal damage, ${ }^{21}$ and the absence of $\beta A P P$ staining in a properly sampled brain with more than three hours' survival after injury would strongly suggest that there has been no significant axonal damage.

Further interpretative difficulties may occur because trauma is not the only cause of disruption of intra-axonal transport of organelles, and failure to appreciate this may lead to over diagnosis of DAI. Work by Sherriff et al ${ }^{2}{ }^{30}$ suggests that white matter pathology in other unrelated conditions may also be associated with staining for $\beta A P P$. It is, therefore, important to exclude other causes before attributing early $\beta A P P$ positivity to trauma. In head injury cases, where there is commonly swelling or shift of the brain secondary to a mass lesion, one of the most usual pathologies to produce $\beta A P P$ staining is secondary ischaemia. ${ }^{19}$ Infarction also impedes axonal transport and for that reason axons round the edge of an ischaemic zone will stain positively with anti- $\beta A P P$, once again well before the cytoplasmic changes of hypoxia can be detected in affected neurons (approximately four to six hours). The question of differentiating between traumatic damage and ischaemia is made more difficult because similar regions tend to be affected: the blood supply to the corpus callosum, the brain stem and indeed some of the deep grey nuclei may be compromised by cerebral swelling and shift, leading to terminal infarction in those areas. In our experience, a wavy pattern of linear staining round an area in the corpus callosum or brain stem tends to be seen in brains showing subfalcine or transtentorial herniation, and we interpret it as indicating early ischaemic rather than traumatic damage. At longer survival times, the distinction between corpus callosum or brain stem infarcts and axonal damage is easily made on standard histological grounds ${ }^{31}$-and it should be remembered that axons in the region of a recent infarct will of course show swellings.

As suggested, inadequate sampling of a brain, particularly one that does not have the macroscopic changes that suggest DAI, ${ }^{17}$ may give a false picture of the degree of axonal damage. Evidence of white matter damage in sites above and below the tentorium is diagnostic of DAI, but how does one interpret axonal injury that is not diffuse according to accepted neuropathological criteria ${ }^{18}$ - for example, only seen in the hemispheres? Milder head injury appears to result in damage to cerebral white matter, without involvement of the brain stem or cerebellum, ${ }^{32}$ and it is probable that such damage has correspondingly milder clinical manifestations than DAI. ${ }^{33}$ A related problem, and not uncommon in the author's experience of a head injury population that includes young cases, and cases of assault, is that there may be evidence of pre-existing axonal damage from an earlier head injury, usually in the form of microglial nodules scattered in hemispheric white matter, although not in the brain stem. The finding of multiple microglial aggregates in white matter is not specific for trauma, although the differential diagnosis is fairly restricted. It includes encephalitis, especially HIV encephalitis, cerebral fat embolism, and a previous episode of global hypoxia. ${ }^{18}$ These conditions should also be excluded before the pathology can be attributed to previous head injury. Once again, such asymptomatic (or apparently asymptomatic) examples of axonal injury presumably represent the clinically and neuropathologically mild end of the spectrum of traumatic brain damage. ${ }^{35}$ It is possible that the descriptions of DAI may need to be modified in the future to include such milder, less widespread forms. This may be helped if quantification methods such as that recently published by Blumbergs et $a l$, proves easily reproducible and clinically reliable. $^{24}$

Finally, the question of timing of the histological changes seen may be an issue in a medicolegal case. The detailed studies of DAI by Adams $e ~_{a l^{17}}{ }^{18}$ using traditional stains, indicated the timing of changes under the broad headings "days", "months", and "years". One would hope that using the more sensitive immunostains for $\beta A P P, P G-M 1$ and GFAP, it would be possible to be more precise. However, as discussed above, experimental studies in animals strongly suggest that the process of axonal reaction to trauma is a process that evolves over the hours (and possibly days) that follow injury. While it is not clear that the process in animals mirrors that in humans, my experience of human DAI suggests that the time course of events is not uniform throughout the same brain. ${ }^{19}$ Under such circumstances, a pathologist would be foolhardy to be drawn into attempting very precise dating of the histological changes.

\section{Summary}

The diagnosis of DAI is not always easy, and should be based on adequate sampling of appropriate anatomical areas from a sliced, fixed brain. It is now recognised that there is a continuum of traumatic white matter damage, and that DAI represents only the severe end of the scale. Such damage may be detected from very shortly after a head injury - a fact that may give rise to some challenging diagnostic problems. Early axonal injury detected by means of $\beta A P P$ immunostaining should be interpreted with caution. The most useful tools currently available for detecting axonal damage are antisera to $\beta A P P, P G-M 1$, and GFAP, used in conjunction with a routine haematoxylin and eosin stain, but even with immunocytochemistry precise dating of histological changes may not be possible. 
1 Povlishock JT, Erb DE, Astruc J. Axonal response to traumatic brain injury: reactive axonal change, deafferentatraumatic brain injury: reactive axonal change, deafferenta-

2 Gentleman SM, Roberts GW, Gennarelli TA, Maxwell WL Adams JH, Kerr S, et al. Axonal injury: a universal consequence of fatal closed head injury? Acta Neuropatho

3 Gennarelli TA. The pathobiology of traumatic brain injury Neuroscientist 1997;33:73-81

4 Pettus EH, Christman CW, Giebel ML, Povlishock JT Traumatically induced altered membrane permeability: its relationship to traumatically induced reactive axonal change. I Neurotrauma 1994;11:507-22.

5 Christman CW, Grady MS, Walker SA, Holloway KL, Povlishock JT. Ultrastructural studies of diffuse axonal injury in humans. $\mathcal{F}$ Neurotrauma 1994;11:173-86.

6 Povlishock JT. Pathobiology of traumatically induced axonal injury in animals and man. Ann Emerg Med 1993;22:9806 .

7 Maxwell WL, Irvine A, Graham DI, Adams JH, Gennarelli TA, Tipperman R, et al. Focal axonal injury: the early axonal response to stretch. $\mathcal{F}$ Neurocytol 1991;20:157-64.

8 Maxwell WL, Watt C, Graham DI, Gennarelli TA Ultrastructural evidence of axonal shearing as a result of lateral acceleration of the head in non-human primates. Acta Neuropathol 1993;86:136-44.

9 Maxwell WL, McCreath BJ, Graham DI, Gennarelli TA. Cytochemical evidence for redistribution of membrane pump calcium-ATPase and ecto-Ca-ATPase activity, and calcium influx in myelinated nerve fibres of the optic nerve after stretch injury. $\mathcal{F}$ Neurocytol $1995 ; 24: 925-42$.

10 Maxwell WL. Histopathological changes at central nodes of Ranvier after stretch-injury. Microsc Res Tech 1996;34:52235 .

11 Maxwell WL, Graham DI. Loss of axonal microtubules after stretch injury. Neuropathol Appl Neurobiol 1996;22:158-9.

12 Povlishock JT. Are the pathobiological changes evoked by traumatic brain injury immediate and irreversible? Brain Pathol 1995;5:415-26.

13 Hammerschlag R, Cyr JL, Brady ST. Axonal transport and the neuronal cytoskeleton. In: Siegel GJ, Agranoff BW, Albers RW, Molinoff PB, eds. Basic neurochemistry. New York: Raven Press, 1994:545-71.

14 Maxwell WL, Povlishock JT, Graham DI. A mechanistic analysis of non-disruptive axonal injury. $\mathcal{F}$ Neurotrauma. [In press.]

15 Pettus EH, Povlishock JT. Characterization of a distinct set of intra-axonal ultrastructural changes associated with traumatically induced alteration in axolemmal permeability. Brain Res 1996;722:1-11.

16 Gean AD. Imaging of head trauma. New York: Raven Press, 1994.

17 Adams JH. Head injury. In: Adams JH, Duchen LW, eds Greenfield's neuropathology. 5th edn. London: Edward Arnold, 1992:106-152.

18 Adams JH, Doyle D, Ford I, Gennarelli TA, Graham DI, McLellan DR. Diffuse axonal injury in head injury: definition diagnosis and grading. Histopathology 1989:15:49-59.

19 Geddes JF, Vowles GH, Beer TW, Ellison DW. The diagno sis of diffuse axonal injury: implications for forensic practice. Neuropathol Appl Neurobiol. [In press.]
20 Gentleman SM, Nash MJ, Sweeting CJ, Graham DI, Roberts GW. Beta-amyloid precursor protein (beta APP) as a marker for axonal injury after head injury. Neurosci Lett 1993;160: 139-44.

21 McKenzie KJ, McLellan DR, Gentleman SM, Graham DI Is beta-APP a marker of axonal damage in short-surviving head injury? Neuropathol Appl Neurobiol 1996;22:160.

22 Sherriff FE, Bridges LR, Sivaloganathan S. Early detection of axonal injury after human head trauma using immunocytochemistry for beta-amyloid precursor protein. Acta Neuropathol 1994;87:55-62.

23 Sherriff FE, Bridges LR, Jackson P. Microwave antigen retrieval of beta-amyloid precursor protein immunoreactivity. Neuroreport 1994;5:1085-8.

24 Blumbergs PC, Scott G, Manavis J, Wainwright H, Simpson DA, McLean AJ. Topography of axonal injury as defined by amyloid precursor protein and the sector scoring method in mild and severe closed head injury. 7 Neurotrauma 1995;12:565-72.

25 Graham DI, Adams JH, Gennarelli TA. Pathology of brain damage in head injury. In: Cooper PR, ed. Head injury. Baltimore: Williams \& Wilkins, 1993:91-113.

26 Bazzaz AA, Bridges LR, Sivaloganathan S. Microglial activation in head injury: an immunohistochemical study using antibodies to CD68 (PG-M1) and CR3/43. Neuropathol Appl Neurobiol 1995;21:446-7.

27 Chesnut RM, Marshall LF, Klauber MR, Blunt BA, Baldwin N, Eisenberg HM, et al. The role of secondary brain injury in determining outcome from severe head injury. F Trauma 1993;34:216-22.

28 Graham DI, Ford I, Adams JH, Doyle D, Teasdale GM Lawrence $\mathrm{AE}$, et al. Ischaemic brain damage is still common in fatal non-missile head injury. $f$ Neurol Neurosurg Psychiatr 1989;52:346-50.

29 Gennarelli TA. Cerebral concussion and diffuse brain injuries. In: Cooper PR, ed. Head injury. 3rd edn. Baltimore: Williams \& Wilkins, 1993:137-58.

30 Raja F, Sherriff FE, Morris CS, Esiri MM. Cerebral axonal damage in HIV infection detected using beta-APP immunocytochemistry. Neuropathol Appl Neurobiol 1996, 22:165.

31 Graham DI. Hypoxia and vascular disorders. In: Adams JH, Duchen LW, eds. Greenfield's neuropathology. 5th edn. London: Edward Arnold, 1992:153-268.

32 Teasdale GM. Head injury. I Neurol Neurosurg Psychiat 1995;58:526-39.

33 Blumbergs PC, Scott G, Manavis J, Wainwright H, Simpson DA, McLean AJ. Staining of amyloid precursor protein to study axonal damage in mild head injury. Lancet 1994;344: study ax

34 Mittl RL, Grossman RI, Hiehle JF, Hurst RW, Kauder DR, Gennarelli TA, et al. Prevalence of MR evidence of diffuse axonal injury in patients with mild head injury and normal head CT findings. A7NR 1994;15:1583-9.

35 Elson LM, Ward CC. Mechanisms and pathophysiology of mild head injury. Semin Neurol 1994;14:8-18. 